\title{
The impact of cultural exposure and message framing on oral health behavior: Exploring the role of message memory
}

\author{
Cameron Brick, M.A. ${ }^{1}$, Scout N. McCully, M.A. ${ }^{2}$, John A. Updegraff, Ph.D. ${ }^{2}$, Phillip J. Ehret, \\ B.A. ${ }^{1}$, Maira A. Areguin, B.A. ${ }^{1}$, and David K. Sherman, Ph.D. ${ }^{1}$ \\ ${ }^{1}$ Psychological \& Brain Sciences, University of California, Santa Barbara, USA \\ ${ }^{2}$ Department of Psychology, Kent State University, USA
}

\begin{abstract}
Background-Health messages are more effective when framed to be congruent with recipient characteristics, and health practitioners can strategically decide on message features to promote adherence to recommended behaviors. We present exposure to United States (U.S.) culture as a moderator of the impact of gain- vs. loss-frame messages. Since U.S. culture emphasizes individualism and approach orientation, greater cultural exposure was expected to predict improved patient choices and memory for gain-framed messages, whereas individuals with less exposure to U.S. culture would show these advantages for loss-framed messages.
\end{abstract}

Methods-223 participants viewed a written oral health message in one of three randomized conditions: gain-frame, loss-frame, or no-message control, and were given ten flosses. Cultural exposure was measured with the proportions of life spent and parents born in the U.S. At baseline and one week later, participants completed recall tests and reported recent flossing behavior.

Results-Message frame and cultural exposure interacted to predict improved patient decisions (increased flossing) and memory maintenance for the health message over one week. E.g., those with low cultural exposure who saw a loss-frame message flossed more. Incongruent messages led to the same flossing rates as no message. Memory retention did not explain the effect of message congruency on flossing.

Limitations-Flossing behavior was self-reported. Cultural exposure may only have practical application in either highly individualistic or collectivistic countries.

Conclusions-In healthcare settings where patients are urged to follow a behavior, asking basic demographic questions could allow medical practitioners to intentionally communicate in terms of gains or losses to improve patient decision making and treatment adherence.

The American Dental Association recommends flossing every day to promote oral health (ADA, 2013), but fewer than half of United States (U.S.) adults floss daily (ADA, 2008). Using printed messages to promote adherence to medical recommendations is a practical and relatively inexpensive intervention to influence health behaviors. Message effectiveness depends on patient characteristics and message content (e.g., Sherman, Uskul, \& Updegraff,

Correspondence concerning this article should be addressed to Cameron Brick, Psychological \& Brain Sciences, University of California, Santa Barbara, Santa Barbara, CA 93106, USA. brickc@ gmail.com. 
2011), but previous work does not provide applied tools to help practitioners determine communication choices in the healthcare setting. Governmental health organizations request that culture be considered in the design of health messages (e.g., Centers for Disease Control, 2008; National Cancer Institute, 2008; National Institute of Dental and Craniofacial Research, 2003), yet it is also unclear how to measure culture within diverse populations for use in message design. The present study makes three advances. First, in an ethnically diverse sample of young U.S. adults we test the effectiveness of framed health messages with a message medium that is easily designed and disseminated in the healthcare context (brochures). Second, we present a new measure of cultural exposure, a construct designed for diverse populations, and examine its interaction with message frame in predicting patient decisions (self-reported flossing). Third, we evaluate the impact of cultural exposure and message frame on the memory for message content, and test whether memory retention is associated with increased health behavior.

\section{Message Frame and Patient Characteristics}

People respond differently to health messages that emphasize gains vs. losses (e.g., Uskul, Sherman, \& Fitzgibbon, 2009; Rothman \& Salovey, 1997). While the prevention vs. detection function of behaviors has a small influence on people's responses to framed messages (Gallagher \& Updegraff, 2012), individual differences of the recipient have a larger influence in predicting the effect of framed messages (Rothman \& Updegraff, 2010; Updegraff, Brick, Emanuel, Mintzer, \& Sherman, 2014). One key characteristic is motivational orientation: how much people are motivated by seeking positive outcomes compared to avoiding negative outcomes (Carver \& White, 1994). Motivational orientation moderates which frame is more effective across a number of health domains, including smoking (Shen \& Dillard, 2009), acceptance of an HPV vaccine (Gerend \& Shepherd, 2007), and oral health behavior (Sherman, Updegraff, \& Mann, 2008). In oral health, relatively approach-oriented people are more persuaded by gain-frame messages (e.g., "If you floss your teeth regularly, you will have healthier teeth and gums") whereas relatively avoidance-oriented people are more persuaded by loss-frame messages (e.g., "If you don't floss regularly, the health of your teeth and gums is at risk"), as shown by increases in flossing behavior, flossing self-efficacy, and intentions to floss (Mann, Sherman, \& Updegraff, 2004; Sherman, Mann, \& Updegraff, 2006).

Cultural background affects cognition, motivation, and emotion at all levels, from basic pattern perception to affective responses of social actions (Heine, Lehman, Markus, \& Kitayama, 1999; Markus \& Kitayama, 1991; Nisbett, Peng, Choi, \& Norenzayan, 2001). Integrating cultural perspectives to improve health communications requires identifying psychological characteristics that vary between cultural groups and examining whether health messages framed to match those characteristics are more effective (Sherman et al., 2011). Although individuals differ in approach and avoidance motivations within countries, the overall balance also varies between cultures (Norenzayan \& Heine, 2005), reflecting differences in chronic motivational orientations (Hamamura \& Heine, 2007; Lee, Aaker, \& Gardner, 2000). For example, people from collectivistic cultures such as Japan are more motivated by avoiding losses than pursuing gains (e.g., Hamamura \& Heine, 2007), perhaps due to the pressure to maintain face (social reputation) and be a valued member of their 
family, groups, and society. Collectivistic individuals are sensitive to losses and vigilant to reputation threats because face is hard to gain but easily lost, and a group's reputation can be easily damaged (Heine et al., 1999). In contrast, members of more individualistic cultures such as the U.S. are more sensitive to gains, individual distinction, and self-promotion (Heine et al., 1999). Framing oral health messages to be congruent with recipient culture leads to higher effectiveness: individualist White British participants indicated more flossing intentions after receiving gain-frame messages, and collectivist British Chinese participants showed more flossing intentions after loss-frame messages (Uskul et al., 2009).

\section{Cultural Exposure}

We focus on a related cultural dimension: the extent to which people are exposed to the U.S., which is relatively individualistic and approach-oriented. We present the novel construct of cultural exposure, the extent to which people are integrated into a host country (in this case, the U.S.), relative to other cultural experiences. We hypothesize that cultural exposure will interact with message frame to determine the effectiveness of persuasive health messages, similar to previous research comparing East-West ethnicities. Two key advantages of the present approach are that cultural exposure can be measured for all ethnic groups and it is practical for use in healthcare settings. The U.S. is highly individualistic and approachoriented (Heine et al., 1999), so we hypothesize that for individuals with greater cultural exposure to the U.S., gain-frame persuasive messages will be congruent and lead to decisions to floss. In contrast, for people with less cultural exposure to the U.S. who are more collectivistic and avoidance-oriented, loss-frame messages will be more effective (Hamamura, Meijer, Heine, Kamaya, \& Hori, 2009; Hofstede, 2001). This prediction should hold in particular for those from Asian and Latino cultures, which form the largest contemporary immigrant groups in the U.S. (Camarota, 2012; Passel \& Cohn, 2012).

Cultural exposure is an objective, demographic measure calculated by the mean of two proportions: life spent in the U.S. and how many of one's parents were born in the U.S. (see Method for calculation). The first component approximates how much an individual has been exposed to U.S. culture in their lifetime relative to other cultures, and the second component indexes how much parental influence stems from U.S. vs. other cultures (Greenfield, 1994). This extends work on generational status (Taylor et al., 2004) that focused on the family level and did not include individual history.

Cultural exposure has advantages compared to previous scales: it does not rely on subjective interpretation or judgments (see Ryder, Alden, \& Paulhus, 2000), and should therefore be less sensitive to situational factors, experimental demand, or reference group effects (a problem where individuals compare themselves to different reference groups in crosscultural research; Heine, Lehman, Peng, \& Greenholtz, 2002). Cultural exposure is easily measured for any individual and is feasible for use in the healthcare context, unlike acculturation scales that must be written to anchor questions between two specific cultures. A recent study examined cultural exposure and message frame congruency using videos that emphasized the importance of flossing, and more flossing over six months was observed for those receiving congruent messages (i.e., high U.S. cultural exposure with gain-frame or low U.S. cultural exposure with loss-frame; Sherman et al., in preparation). 
The present study advances beyond previous work by 1) testing the effectiveness of an easily disseminated printed message; 2) examining the effect of congruency on memory retention and the relation between memory and behavior; and 3) evaluating the congruency effect on oral health behavior over one week among young adults who are provided flosses.

\section{Hypotheses}

The first goal is to evaluate whether framed health messages are differentially effective for individuals based on their cultural exposure.

H1: Gain-frame messages will increase flossing among individuals high in cultural exposure to the U.S., and loss-frame messages will increase flossing among individuals low in cultural exposure to the U.S., compared to incongruent messages and no message.

The second goal is to evaluate memory as both an outcome and a mechanism of the key effect in H1 (as suggested by Mann et al., 2004). Memory represents what information is available for cognition over time, so memory is a strong candidate for causing the heightened effectiveness of congruent health messages. For example, the MODE model (Fazio \& Towles-Schwen, 1999) posits that attitudes readily accessible from memory will better predict behavior than less accessible attitudes. Also, health messages that are framed to be congruent with approach-avoidance orientation are processed more systematically (Rothman \& Updegraff, 2010). Individuals elaborate more on congruently framed messages, and message effectiveness is higher when congruently framed messages present strong evidence (Updegraff, Sherman, Luyster, \& Mann, 2007). Therefore, we expect that participants who receive congruent (vs. incongruent) health messages will have better recall (see Higgins \& Tykocinski, 1992 on recall and health messages). In line with this claim, memory recall was improved for culturally congruent messages in two non-health studies (Hamamura et al., 2009).

H2: Individuals high in cultural exposure to the U.S. who receive a gain-frame message, and individuals low in cultural exposure to the U.S. who receive a loss-frame message, will better remember the health message compared to the incongruent message.

The third goal is to test whether increased memory for congruent messages leads to an improvement in health behavior. Because cultural congruency is expected to increase engaged information processing and elaboration, it could also lead to the increased accessibility of flossing-related thoughts and cause an increase in flossing behavior.

H3: The cultural congruency effect on flossing will be mediated by increased memory retention.

\section{Methods}

\section{Participants}

Two hundred twenty-three undergraduates participated for course credit, $M(S D)$ age $=20.1(3.29)$ years; $62.8 \%$ female; $41.3 \%$ Caucasian, $17.4 \%$ Latino American, $15.7 \%$ 
African American, 15.7\% Asian American, 1.4\% Native American, 7.2\% other ethnicity, and $1.3 \%$ declined to answer (see Table 1). Recruitment occurred at two universities, one Midwestern and one Western, to increase ethnic and cultural diversity. The study was approved by the Human Subjects Review Boards of both universities. Participants were randomly assigned to condition: gain-frame ( $n=75)$, loss-frame $(n=76)$, or no message control ( $n=72)$. U.S. cultural exposure varied from .002-1, $M(S D)=0.73(0.34)$. At baseline (in the laboratory) and during the one-week follow-up (online), participants reported their flossing behavior and completed recall memory tests on a computer. At the conclusion of the first session, participants were given ten individually wrapped flosses to enable flossing regardless of home supplies.

\section{Measures and Stimuli}

Oral health messages-Two oral health brochures were developed (gain-frame: 628 words, loss-frame: 646 words) to convince individuals to brush and floss their teeth (see Supplementary Materials). Each black-and-white tri-fold brochure had a professional layout with images and text (with information adapted from American Dental Association, 2013), and each was ostensibly produced by the American Dental Society as indicated by several logos. The brochure contained six sections: "Your Teeth," "Your Breath," "Plaque," "Your Gums," "Your Cardiovascular System," and "How to Floss Correctly." Across brochure types, the same content was presented with different frames. For example, the gain brochure read: "A consistent routine of brushing and flossing maintains the health of your teeth and gums," while the loss brochure read: "An inconsistent routine of brushing and flossing hurts the health of your teeth and gums." Each brochure contained 18 framed statements and 20 unframed statements, e.g., "To floss your teeth, take about 18 inches of floss in your hand." Participants were invited to keep the brochure.

Recall-Following a 10-minute figure-drawing delay task (adapted from Christianson, Loftus, Hoffman, \& Loftus, 1991), participants took a surprise recall memory test and were asked to type all the statements they remembered, one per box. Eighteen boxes were provided, and participants made a substantive effort, spending $M(S D)=5.92(3.73)$ minutes on free recall (recorded with survey software). Next, participants were shown the six section headers and were provided five blank boxes per cue for additional cued recalls.

Recall coding-Two trained coders, unaware of condition or participant characteristics, coded each recalled statement for accuracy, frame, and the matching brochure statement (statement ID). Recalls were coded as accurate when they retained accurate facts. Recall frame was coded as gain, loss, unframed (if the original statement was framed, but the recall was not), or control. Statement ID allowed the removal of duplicate recalls. For recalls that combined statements, credit was given for both statements when coders agreed on accuracy, frame, and statement ID for both. Coding reliability (intraclass correlation) for statement ID was very good, baseline $r=.93$, follow-up $r=.90$; reliability was also good for frame, baseline $r=.80$, follow-up $r=.87$. Accuracy was rated dichotomously, and most recalls were rated "accurate" (94\%). Cohen's kappa is typically used to assess inter-rater reliability with dichotomous ratings, but it has well-known problems for uneven category base rates. Therefore, Gwet's AC1 (Gwet, 2002) was used to calculate inter-rater reliability for the 
accuracy, and inter-rater reliability was also good (baseline $\mathrm{AC} 1=.93$; follow-up $\mathrm{AC} 1=.87$ ). An independent third coder, unaware of condition or participant characteristics, resolved all discrepancies. Correct recalls from the free and cued tests were summed for overall recall frequency and accuracy. See Table 1.

Cultural exposure-Participants were asked: "How long have you lived in the United States? (in years)." Years were divided by age to yield the proportion of life spent in the U.S. Then, participants were asked: "How many of your parents were born in the United States?" $(0,1$, or 2$)$. This value was divided by two so that both components reflected a proportion from 0 (low exposure to U.S. culture) to 1 (high exposure). The components were correlated $r=.48$, and were averaged together to yield cultural exposure. By ethnicity, Caucasians had the highest cultural exposure to the U.S.: $M(S D)=.93(.19)$, followed by African Americans: . 88(.27), Latino Americans: .58(.20), Asian Americans: .51(.28), and Other: .42(.38). See Table 1.

Motivational orientation-The Behavioral Inhibition and Behavioral Approach Scales (Carver \& White, 1994) measured approach orientation (BAS; Cronbach's $a=.80$ ) and avoidance orientation (BIS; $a=.75$ ). Motivational orientation (MO) was calculated by $(\mathrm{MO}=\mathrm{BAS}-\mathrm{BIS})$, such that $\mathrm{MO}>0$ represented a relative approach orientation (Mann et al., 2004; Sherman et al., 2006). Cultural exposure was uncorrelated with MO, is BAS=.04, $\mathrm{BIS}=.04, \mathrm{MO}=.00, p \mathrm{~s} \geq 36$, consistent with a recent study (Sherman et al., in preparation). See Discussion.

Demographics-Participants reported demographics including age, gender, and ethnicity, for which participants were asked: "What is your racial/ethnic background?" and selected from: White/Caucasian, African American, Asian American, Hispanic/Latino, Native American, and Other. See Table 1.

Previous flossing-Past flossing behavior was measured before the manipulation with a single item (Davidson, Rams, \& Andersen, 1997): "In general, how often do you floss your teeth?”, measured from 1 (Never) to 7 (Two or more times per day). See Table 1.

Additional measures-Participants completed measures assessing additional constructs, including brushing frequency (one question); flossing self-efficacy, perceived value, and enjoyment (10 questions); flossing intentions (one question); individualism (16 questions); and perceived susceptibility for oral health problems (five questions; see Supplementary Analyses). There were no significant correlations with cultural exposure nor any main effects or interactions with these variables, and they are not discussed further.

Follow-up: Self-reported flossing and recall-After one week, participants received a study reminder by email, were contacted by phone after three days if they had not responded, and completed a questionnaire online, followed by a debriefing on the purpose of the study. Nine participants (4\%) failed to complete the follow-up. Participants first reported how many times in the past week they flossed their teeth, measured by integers between 0 and "14+," in addition to an additional item measuring brushing frequency (asking about brushing may also reduce demand characteristics on the flossing measure). Participants then 
repeated the free and cued recall tests from baseline. Finally, participants also completed a measure of intentions to schedule a dental appointment. There were no significant main effects or interactions with intentions.

Sampling method-In the interest of transparent sampling methods (Simmons, Nelson, \& Simonsohn, 2011), all measures and conditions are described above, and all sampled participants are described. The first data collection period $(n=154)$ lasted 10 weeks (one quarter). This was inadequate for the desired statistical power, so collection was extended for the remainder of the following quarter $(n=79)$. Confirmatory hypotheses were tested after data collection ended.

Analytic plan-H1 was evaluated with a hierarchical linear regression predicting flossing behavior from frame and cultural exposure (CE), with motivational orientation and its interaction with frame entered in the second step to isolate the unique contribution of CE. To highlight practical implications, a logistic regression further evaluated who flossed at recommended rates between individuals who received a matching, mismatching, or no message. $\mathrm{H} 2$ involves memory retention across one week and was tested with generalized estimating equations (GEE), an extension of the generalized linear model for regressions with repeated outcomes that allows for correlated outcomes and reduces Type I errors compared to using separate regressions for each outcome period. GEE was used to predict memory retention over time as a function of $\mathrm{CE}$ and message frame. $\mathrm{H} 3$ was evaluated with a Sobel-Goodman test of memory decay mediating the effect of message congruency on flossing. Age, gender, and ethnicity (dummy-coded with the most numerous group, Caucasian, as reference) have previously predicted flossing behavior (e.g., Updegraff et al., 2014), so these variables were retained as covariates in each analysis to avoid cohort effects and better isolate the effect of CE.

\section{Results}

\section{Self-reported flossing}

First, we examined whether receiving a congruent (vs. incongruent) message led to increased self-reported flossing. A hierarchical linear regression predicted flossing from frame condition and cultural exposure (CE; continuous) with age, gender, ethnicity, and previous flossing as covariates (see Table 2). In Step 1, prior flossing was the only significant covariate, $B=.58, S E=.08, p<.001$. Two main effects emerged. First, individuals who read a loss-frame message, $M(S D)=5.64(.37)$, reported flossing more during the week than those who read a gain-frame message, $M(S D)=5.16(.37) ; B=4.46, S E=1.61, p=.006$. Second, low-CE individuals flossed more, $M(S D)=6.23(.47)$, compared to high-CE, $M(S D)=4.87(.36) ; B=-4.98, S E=1.58, p=.002$. These main effects were qualified by the predicted two-way interaction, $B=5.10, S E=1.90, p=.008, \eta^{2}=.06$ (see Figure 1). CE was entered as a continuous variable, and because $\mathrm{CE}$ was peaked at 1 (54.7\% of cases) and ranged down to .002, contrasts were created at high CE (1) and low CE (the average of non-1 values: .45). High-CE participants who received a gain-frame message flossed somewhat more, $M(S D)=5.20(.50)$, than those who received a loss-frame message, $M(S D)=4.55(.48) ; B=-.93, S E=.66, p=.16$. Low-CE participants who saw a loss-frame 
message flossed significantly more, $M(S D)=7.29(.67)$, compared to those who saw a gainframe message, $M(S D)=5.13(.59) ; B=13.9, S E=3.48, p<.001$.

In sum, $\mathrm{H} 1$ was supported, especially among low-CE individuals, with a directional but not significant contrast among high-CE individuals. To examine whether this interaction was driven by motivational orientation (MO), in the next regression step MO and its interaction with message frame were included as predictors. There was no main effect, nor was there a significant interaction of $\mathrm{MO}$ with frame, $p \mathrm{~s} \geq 31$. As hypothesized, the interaction between $\mathrm{CE}$ and frame remained significant, $B=4.97, S E=1.92, p=.01, \eta^{2}=.05$ (see Table 2).

Because there is an objective threshold of recommended flossing (daily), the next analysis predicted who was flossing at recommended levels. Additionally, we compared those who were in a congruent condition (i.e., randomly assigned to a condition that "matched" or was congruent with their measured levels of cultural exposure) and those who were in an incongruent condition to those who received no message. Those who were in congruent conditions ( $n=75$ ) were either those with $C E \leq 45$ who received a loss-framed message, or those with $\mathrm{CE}=1$ who received a gain-framed message; the remaining participants saw incongruent messages $(n=76)$. In a logistic regression using age, gender, ethnicity, and prior flossing as covariates, a congruency effect was observed on flossing at recommended rates, $\chi^{2}(7)=55.6, p<.001$. Planned contrasts showed that participants who received a congruent message were more likely to floss at recommended rates, $44.0 \%, S E=4.86$, than those who read an incongruent message, $28.4 \%, S E=4.45$, odds ratio $(O R)=2.94, S E=1.43, p=.03$. Incorporating the control condition, congruent participants were also more likely to floss at recommended levels, $44.3 \%, S E=5.03$, than those in the incongruent and no-message groups combined, 30.7\%, $S E=3.27 ; O R=2.39, S E=0.93, p=.03$. In addition, the likelihood of flossing at recommended levels was the same between incongruent participants, 29.6\%, $S E=4.37$, and control, 34.4\%, $S E=4.38 ; O R=0.68, S E=0.34, p=.44$.

\section{Statement memory}

Memory for the health message was measured at baseline and after one week, enabling the test of memory retention for the health message. Using GEE (see Analytic Plan), no main effects were significant, $p s \geq 13$. In order to probe interactions, the dummy variable of ethnicity was dropped (Dawson \& Richter, 2006). There was an unpredicted two-way interaction between time and frame, $B=-2.52, S E=1.08, p=.02$, reflecting that loss-frame participants had more memory decay between baseline, $M(S E)=6.83(.27)$ statements recalled, and follow-up, $M(S E)=4.22(.27)$, than gain-frame participants, baseline $M(S E)=6.89(.27)$, and follow-up $M(S E)=4.69(.28)$.

This effect was qualified by the predicted three-way interaction of frame, cultural exposure, and time, $B=3.98, S E=1.35, p=.003$ (see Figure 2). For high-CE individuals, memory decayed over time for both gain- (slope $\neq$ zero $B=-1.49, S E=.44, p=.001$ ) and loss-frame (slope $\neq$ zero $B=-3.23, S E=.42, p<.001$ ) conditions, and memory decayed more in the lossthan gain-frame condition (difference in simple slopes $B=1.74, S E=.61, p=.004$ ). For low$\mathrm{CE}$ individuals, memory decayed over time for both gain- (slope $\neq$ zero $B=-2.83, S E=.38$, $p<.001$ ) and loss-frame (slope $\neq$ zero $B=-2.05, S E=.45, p<.001$ ) conditions, and the slope representing decline in memory was steeper for gain-frame although not significantly, $B=-$. 
78, $S E=.59, p=.19$. In sum, $\mathrm{H} 2$ received support among high-CE individuals, who retained better memory over one week after a gain-frame message, and directional (but not significant) support among low-CE individuals who received a loss-frame message.

\section{Mediation}

Memory was unrelated to self-reported flossing, so H3 was rejected. Using age, gender, ethnicity, and prior flossing as covariates in a Sobel-Goodman test, memory decay did not predict flossing, $B=.39, S E=.72, p=.58$. The interaction of message frame and cultural exposure had independent effects on memory and self-reported flossing.

\section{Discussion}

This study provides three advances in understanding persuasive communication for culturally diverse populations. First, this study explored a message medium (brochure) that is typical for the healthcare context being studied (e.g., dentist offices) and can be easily adapted and distributed by healthcare professionals. Second, it introduced the novel, practical index of cultural exposure (CE) and showed that strategically matching culture to message frame can improve patient decisions about oral health (H1) and message memory for health recommendations (H2). Third, it demonstrated that the content of congruent health messages are better remembered, and showed that recall does not explain the behavior effect (H3).

The hypotheses were generally supported, although the congruency effect on flossing was driven more by individuals with low $\mathrm{CE}$ (H1) and the congruency effect on memory was driven more by individuals with high CE (H2). Participants who received an incongruent message flossed equivalently to participants who received no message, which reinforces the importance of considering patient characteristics during communication. A key advance was finding the congruency effects with a simple, demographic measure of culture in an ethnically diverse sample. Dental disease is largely preventable, but not all groups within the U.S. have the information or ability to engage in oral health promotion (U.S. Department of Health and Human Services, 2000). The inclusion of African Americans, Latino Americans, and Asian Americans extends the generalizability of cultural congruency findings, and identifying effective messages for these groups is a step towards addressing racial and ethnic oral health disparities (National Institute of Dental and Craniofacial Research, 2003).

There was a strong advantage of loss-frame messages among individuals low in U.S. CE. A loss-frame advantage on behavior was also shown among a large sample of U.S. adults (Sherman et al., in preparation). This loss-frame advantage challenges long-standing beliefs about how health messages should be framed to encourage adherence to preventative health behaviors such as flossing. A recent meta-analysis showed that gain-frame messages are slightly more effective than loss-frame messages for encouraging preventative behaviors in general (Gallagher \& Updegraff, 2012). However, past research in message framing has been conducted almost exclusively in individualistic countries: a meta-analysis reviewed 95 studies and found that only one sample was from a collectivistic country (Taiwan; Gallagher \& Updegraff, 2012). Our results suggest that loss-frame messages may be more effective 
than gain-frame messages for individuals low in U.S. CE, and for collectivistic populations (cf., Pakpour, Yekaninejad, Sniehotta, Updegraff, \& Dombrowski, 2014).

It would be valuable for future research to identify the psychological and sociological correlates of U.S. CE as measured here. Motivational orientation (as assessed by BIS-BAS measure) appears unrelated, as it was not correlated with CE and it did not mediate the cultural congruency effect in this study. To examine the sociological correlates of CE, we recently completed a feasibility pilot to evaluate the logistics of delivering framed health messages in dental clinics and offices in central California. A validated scale of U.S. acculturation of Hispanics was administered to participants who spoke at least some Spanish (Marin, Sabogal, Marin, Otero-Sabogal, \& Perez-Stable, 1987). CE was highly correlated in this sample with Hispanic acculturation at $r(32)=.62, p<.001$, demonstrating convergent validity for CE. Recall that the behavior effect in the present study was driven by a lossframe advantage for individuals with low CE. Taken together, it is possible that the key construct behind this effect is not exposure to U.S. culture but exposure to a common feature of the other cultures in our sample (e.g., collectivism, as the majority of low CE participants were from collectivistic cultures, reflecting the most numerous immigrant groups to the U.S.; see Camarota, 2012).

Other psychological variables related to motivation may be associated with CE. Prior work using prevention/promotion as an index of MO (Lockwood, Jordan, \& Kunda, 2002) found that regulatory focus, an alternative construct of individual difference motivation to BISBAS, mediated the culture by frame interaction on flossing intentions (Uskul et al., 2009). Therefore, measures of regulatory focus may be better suited than BIS-BAS to capture individual differences related to the exposure to the U.S. relative to other cultures. The construct of cultural exposure will benefit from further examinations in diverse field settings that include assessment of individual differences such as acculturation and prevention/ promotion focus.

The current study also found that message congruency led to improved memory retention across one week, and tested whether message memory was a mediator of the congruency effect on flossing behavior. Despite the logic for how memory drives behavior, there are several possible explanations for the lack of mediation. First, memory might not be a mechanism of the congruency effect; congruency may lead to a cascade of effects on cognition and behavior. Second, behavior might be driven less by memory for the content of the message than the overall memorability of the health message. The third explanation is operational. As a self-report measure, successful memory recall requires conditions beyond the presence of underlying semantic memory such as task motivation and conscious processing. Other types of memory tests that rely less on these conditions (e.g., recognition tests) may yet reveal associations with behavior.

\section{Limitations}

First, although interactions between CE and message frame predicting oral health behavior have been observed in two samples (the present study and Sherman et al., in preparation), the effectiveness of $\mathrm{CE}$ as a moderator may be dependent upon sample features. The current study was conducted in the U.S., which is highly individualistic, and the low-CE individuals 
mostly came from relatively collectivistic cultures (i.e., East Asia and Latin America)., CE may be particularly useful for determining the optimal message frame in countries that are either highly individualistic or collectivistic, and where the largest immigrant groups differ on these dimensions. CE may be less informative for a recent U.S. immigrant from Northern Europe, a relatively individualistic region. CE may also be a poor measure for Native Americans, who have high exposure to the U.S. but are generally collectivistic (Fryberg \& Markus, 2003). For these groups we suggest consulting other framing moderators such as perceived susceptibility (Updegraff et al., 2014).

Second, it is unclear based on the present research how to optimally frame medical communications for individuals with moderate CE. Again, we suggest researchers look to other established moderators (see Updegraff \& Rothman, 2013 for discussion). Third, the current measure of ethnicity was confounded with race and did not separate White and nonWhite Hispanics. Fourth, self-reported flossing behavior could have introduced measurement error through social desirability bias or inaccurate memory, although this would not explain the observed interaction patterns.

\section{Conclusions}

Cultural exposure is simple to measure, calculate, and use in healthcare contexts, and can predict differential message frame effectiveness in a sample of diverse U.S. citizens. These findings inform medical decision making in two ways. First, health practitioners can use strategic communication to improve message effectiveness (e.g., Abhyankar, Summers, Velikova, \& Bekker, 2014). In healthcare settings where patients are urged to perform (or not perform) a behavior, asking two questions about cultural exposure would allow medical practitioners to strategically describe the consequences of a behavior primarily in terms of gain or loss. Second, patients who receive congruent messages appear to decide on better health behaviors. In practice, cultural exposure could be measured at patient intake and used to inform provider communication during the patient session.

Being aware of cultural characteristics can help practitioners promote oral health behaviors in diverse populations. Cultural congruency leads to improved memory as well as greater adherence, and these two effects appear independent. Almost any health suggestion can be framed in terms of gains or losses, and framing can be employed in any medium, such as speech, text, or video. We encourage oral health professionals to adapt and use our brochures, and hope that sharing these materials informs the development of effective health messages in other health areas. In sum, strategic framing choices in health communication can improve patient decisions and behaviors.

\section{Acknowledgments}

We thank Jacob S. Brookfield for assistance with data analysis, and David K. Sherman's research group for helpful comments.

Financial support for this study was provided in part by grants from the National Science Foundation (DGE-0707430) and the National Institute of Dental and Craniofacial Research (R21-DE019704-01). The funding agreements ensured the authors' independence in designing the study, interpreting the data, writing, and publishing the report. 


\section{References}

Abhyankar P, Summers BA, Velikova G, Bekker HL. Framing options as choice or opportunity: Does the frame influence decisions? Medical Decision Making. 2014; 34(5):567-582. DOI: 10.1177/0272989X14529624 [PubMed: 24732048]

American Dental Association. The public speaks up on oral health care: an ADA and Crest/Oral-B survey. GFK Roper Public Affairs \& Media; 2008. Retrieved from www.crest.com/ada-webcast/ surveyfindings.pdf

American Dental Association. Flossing. 2013. Retrieved October 16, 2014, from http:// www.mouthhealthy.org/en/az-topics/f/flossing

Camarota, SA. Immigrants in the United States, 2010: A profile of America's foreign-born population. 2012 Jul 20. Retrieved June 16, 2014, from http://cis.org/2012-profile-of-americas-foreign-bornpopulation

Carver CS, White TL. Behavioral inhibition, behavioral activation, and affective responses to impending reward and punishment: The BIS/BAS scales. Journal of Personality and Social Psychology. 1994; 67:319-333.

Centers for Disease Control. Promoting healthy parenting practices across cultural groups: A CDC research brief. 2008. Retrieved from http://safesupportivelearning.ed.gov/resources/promotinghealthy-parenting-practices-across-cultural-groups-cdc-research-brief

Christianson S-Å, Loftus EF, Hoffman H, Loftus GR. Eye fixations and memory for emotional events. Journal of Experimental Psychology: Learning. 1991; 17(17):693-701. DOI: 10.1037/0278-7393.17.4.693

Davidson PL, Rams TE, Andersen RM. Socio-behavioral determinants of oral hygiene practices among USA ethnic and age groups. Advances in Dental Research. 1997; 11(2):245-253. [PubMed: 9549990]

Dawson JF, Richter AW. Probing three-way interactions in moderated multiple regression: Development and application of a slope difference test. Journal of Applied Psychology. 2006; 91(4): 917-926. DOI: 10.1037/0021-9010.91.4.917 [PubMed: 16834514]

Fazio, RH.; Towles-Schwen, T. The MODE model of attitude-behavior processes. In: Chaiken, S.; Trope, Y., editors. Dual process theories in social psychology. New York: Guilford Press; 1999. p. 97-116.

Fryberg SA, Markus HR. On being American Indian: Current and possible selves. Self and Identity. 2003; 2(4):325-344. DOI: 10.1080/714050251

Gallagher KM, Updegraff JA. Health message framing effects on attitudes, intentions, and behavior: a meta-analytic review. Annals of Behavioral Medicine: A Publication of the Society of Behavioral Medicine. 2012; 43(1):101-116. DOI: 10.1007/s12160-011-9308-7 [PubMed: 21993844]

Gallagher KM, Updegraff JA, Rothman AJ, Sims L. Perceived susceptibility to breast cancer moderates the effect of gain- and loss-framed messages on use of screening mammography. Health Psychology: Official Journal of the Division of Health Psychology, American Psychological Association. 2011; 30(2):145-152. DOI: 10.1037/a0022264

Gerend MA, Shepherd JE. Using message framing to promote acceptance of the human papillomavirus vaccine. Health Psychology. 2007; 26:745-752. [PubMed: 18020847]

Greenfield, PM. Independence and interdependence as developmental scripts: Implications for theory, research, and practice. In: Greenfield, PM.; Cocking, RR., editors. Bridging Cultures, Readings 4bk Set: Cross-Cultural Roots of Minority Child Development. 1. Hillsdale, N.J: Psychology Press; 1994.

Gwet, K. Kappa statistic is not satisfactory for assessing the extent of agreement between raters. Gaithersburg, MD: STATAXIS Consulting; 2002.

Hamamura, T.; Heine, SJ. Approach and avoidance motivations across cultures. In: Elliot, AJ., editor. Handbook of approach and avoidance motivations. Mahwah, NJ: Erlbaum; 2007.

Hamamura T, Meijer Z, Heine SJ, Kamaya K, Hori I. Approach-avoidance motivation and information processing: cross-cultural analysis. Personality and Social Psychology Bulletin. 2009; 35(4):45462. [PubMed: 19164704] 
Heine SJ, Lehman DR, Markus HR, Kitayama S. Is there a universal need for positive self-regard? Psychological Review. 1999; 106:766-794. [PubMed: 10560328]

Heine SJ, Lehman DR, Peng K, Greenholtz J. What's wrong with cross-cultural comparisons of subjective Likert scales?: The reference-group effect. Journal of Personality and Social Psychology. 2002; 82(6):903-918. [PubMed: 12051579]

Higgins ET, Tykocinski O. Self-discrepancies and biographical memory: Personality and cognition at the level of psychological situation. Personality and Social Psychology Bulletin. 1992; 18(5):527535. DOI: $10.1177 / 0146167292185002$

Hofstede, G. Culture's Consequences: Comparing Values, Behaviors, Institutions and Organizations Across Nations. 2. Thousand Oaks, Calif: SAGE Publications, Inc; 2001.

Lee AY, Aaker JL, Gardner WK. The pleasures and pains of distinct self-construals: the role of interdependence in regulatory focus. Journal of Personality and Social Psychology. 2000; 78:1122-1134. [PubMed: 10870913]

Lockwood P, Jordan CH, Kunda Z. Motivation by positive and negative role models: Regulatory focus determines who will best inspire us. Journal of Personality and Social Psychology. 2002; 83:854864. [PubMed: 12374440]

Mann TL, Sherman DK, Updegraff JA. Dispositional motivations and message framing: A test of the congruency hypothesis in college students. Health Psychology. 2004; 23:330-334. [PubMed: 15099176]

Marin G, Sabogal F, Marin BV, Otero-Sabogal R, Perez-Stable EJ. Development of a short acculturation scale for Hispanics. Hispanic Journal of Behavioral Sciences. 1987; 9(2):183-205. DOI: $10.1177 / 07399863870092005$

Markus HR, Kitayama S. Culture and the self: Implications for cognition, emotion, and motivation. Psychological Review. 1991; 98(2):224.

National Cancer Institute. Making health communication programs work. National Institutes of Health; 2008. Retrieved from http://www.cancer.gov/cancertopics/cancerlibrary/pinkbook/page6

National Institute of Dental and Craniofacial Research. A plan to eliminate craniofacial, oral, and dental health disparities. 2003.

Nisbett RE, Peng K, Choi I, Norenzayan A. Culture and systems of thought: Holistic versus analytic cognition. Psychological Review. 2001; 108(2):291-310. doi:http:// dx.doi.org.proxy.library.ucsb.edu:2048/10.1037/0033-295X.108.2.291. [PubMed: 11381831]

Norenzayan A, Heine SJ. Psychological universals: What are they and how can we know? Psychological Bulletin. 2005; 131(5):763-784. [PubMed: 16187859]

Pakpour AH, Yekaninejad MS, Sniehotta FF, Updegraff JA, Dombrowski SU. The effectiveness of gain- versus loss-framed health messages in improving oral health in Iranian secondary schools: A cluster-randomized controlled trial. Annals of Behavioral Medicine. 2014; 47(3):376-387. DOI: 10.1007/s12160-013-9543-1 [PubMed: 24163187]

Passel, JS.; Cohn, D. U.S. foreign-born population: How much change from 2009 to 2010?. 2012. Retrieved from http://www.pewhispanic.org/2012/01/09/u-s-foreign-born-population-how-muchchange-from-2009-to-2010/

Rothman AJ, Salovey P. Shaping perceptions to motivate healthy behavior: The role of message framing. Psychological Bulletin. 1997; 121:3-19. [PubMed: 9000890]

Rothman, AJ.; Updegraff, JA. Specifying when and how gain- and loss-framed messages motivate healthy behavior: An integrated approach. In: Keren, G., editor. Perspectives on Framing. London: Psychology Press / Taylor \& Francis; 2010. p. 257-278.

Ryder AG, Alden LE, Paulhus DL. Is acculturation unidimensional or bidimensional? A head-to-head comparison in the prediction of personality, self-identity, and adjustment. Journal of Personality and Social Psychology. 2000; 79(1):49-65. [PubMed: 10909877]

Shen L, Dillard JP. Message frames interact with motivational systems to determine depth of message processing. Health Communication. 2009; 24(6):504-514. DOI: 10.1080/10410230903104897 [PubMed: 19735028]

Sherman, DK.; Brick, C.; Binning, KR.; Mintzer, RE.; McCully, SN.; Updegraff, JA. Culture and health communication: The role of cultural exposure in health message framing. (in preparation) 
Sherman DK, Mann TL, Updegraff JA. Approach/avoidance motivation, message framing, and health behavior: understanding the congruency effect. Motivation and Emotion. 2006; 30(2):165-169. DOI: 10.1007/s11031-006-9001-5 [PubMed: 19079797]

Sherman DK, Updegraff JA, Mann T. Improving oral health behavior: A social psychological approach. The Journal of the American Dental Association. 2008; 139(10):1382. [PubMed: 18832274]

Sherman DK, Uskul AK, Updegraff JA. The role of the self in responses to health communications: A cultural perspective. Self and Identity. 2011; 10:284-294. [PubMed: 21980260]

Simmons JP, Nelson LD, Simonsohn U. False-positive psychology: Undisclosed flexibility in data collection and analysis allows presenting anything as significant. Psychological Science. 2011; 22(11):1359-1366. DOI: 10.1177/0956797611417632 [PubMed: 22006061]

Taylor SE, Sherman DK, Kim HS, Jarcho J, Takagi K, Dunagan MS. Culture and social support: who seeks it and why? Journal of Personality and Social Psychology. 2004; 87(3):354-362. DOI: 10.1037/0022-3514.87.3.354 [PubMed: 15382985]

Updegraff JA, Brick C, Emanuel A, Mintzer RE, Sherman DK. Message framing for health: Moderation by perceived susceptibility and motivational orientation in a diverse sample of Americans. Health Psychology. 2014

Updegraff JA, Rothman AJ. Health message framing: Moderators, mediators, and mysteries. Social and Personality Psychology Compass. 2013; 7(9):668-679. DOI: 10.1111/spc3.12056

Updegraff JA, Sherman DK, Luyster FS, Mann TL. Understanding how tailored communications work: The effects of message quality and congruency on perceptions of tailored health communications. Journal of Experimental Social Psychology. 2007; 43:248-256.

U.S. Department of Health and Human Services. Oral health in America: A report of the Surgeon General (executive summary). Rockville, MD: U.S. Department of Health and Human Services, National Institute of Dental and Craniofacial Research, National Institutes of Health; 2000.

Uskul AK, Sherman DK, Fitzgibbon J. The cultural congruency effect: Culture, regulatory focus, and the effectiveness of gain- vs. loss-framed health messages. Journal of Experimental Social Psychology. 2009; 45(3):535-541. 


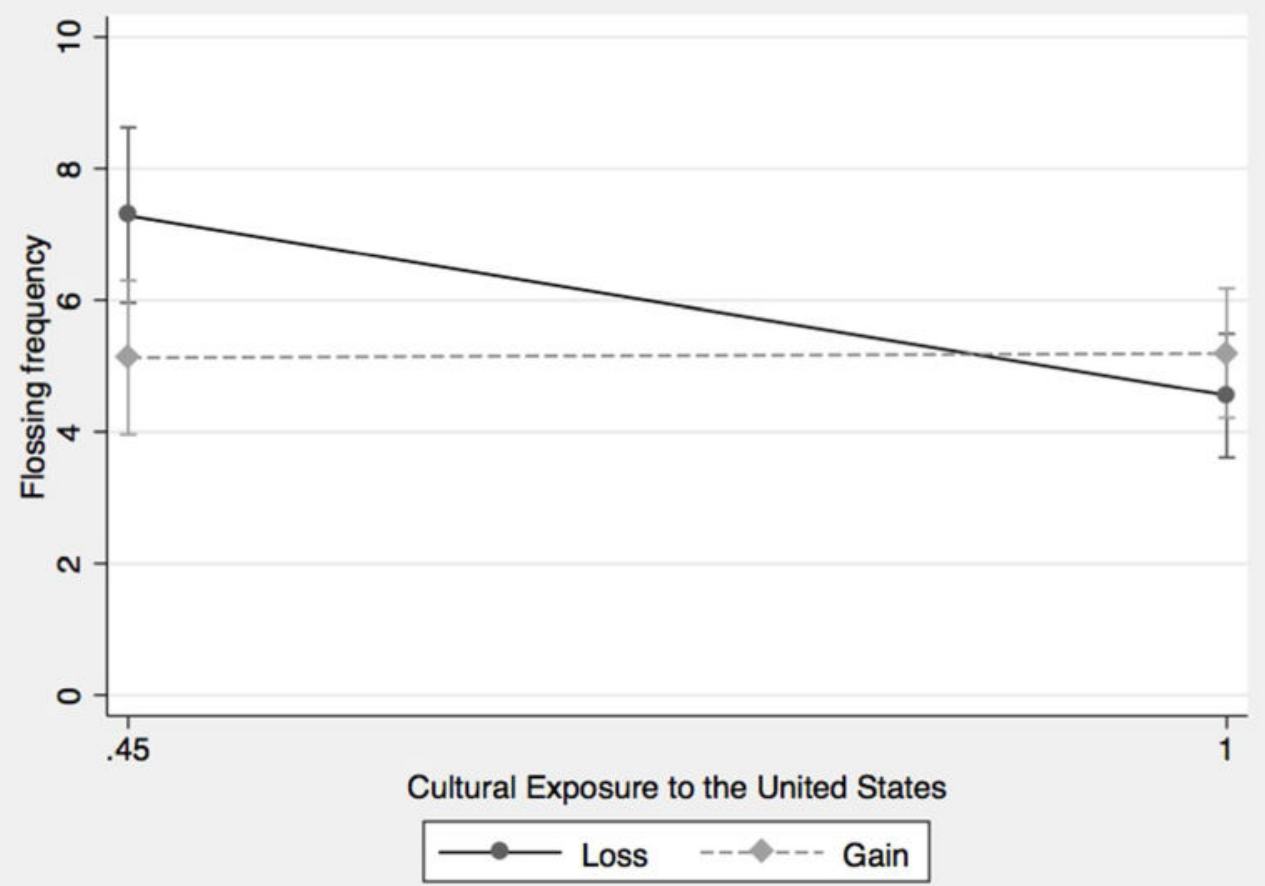

Figure 1.

Self-reported flossing behavior by cultural exposure and message frame, with age, gender, ethnicity, and previous flossing as covariates (Step 1 in Table 2). 

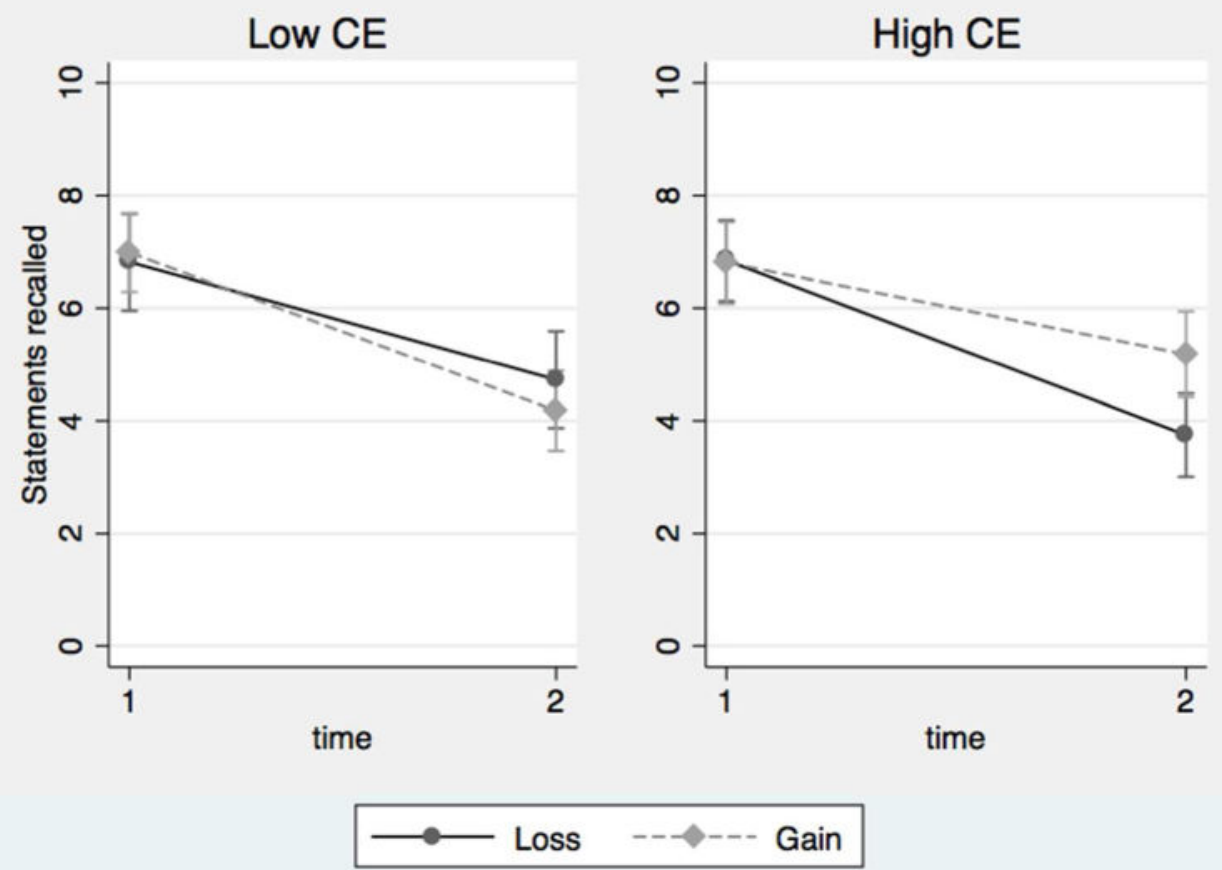

Figure 2.

Memory decay as a function of cultural exposure, message frame, and time, with age, gender, ethnicity, and previous flossing as covariates (Step 1 in Table 2). 


\section{Table 1}

Demographic and analytic variables by condition.

\begin{tabular}{lccc}
\hline$N=\mathbf{2 2 3}$ & Gain & Loss & No message \\
\hline & \multicolumn{3}{c}{$N$} \\
\hline Total & 75 & 76 & 72 \\
\hline Female & 48 & 50 & 42 \\
Caucasian & 30 & 30 & 32 \\
African American & 13 & 12 & 10 \\
Latino American & 14 & 12 & 13 \\
Asian American & 9 & 14 & 12 \\
Other & 9 & 8 & 5 \\
\hline & & $M(S D)$ & \\
\hline Age & $20.1(3.29)$ & $19.7(1.45)$ & $19.5(1.40)$ \\
Cultural exposure & $.73(.34)$ & $.77(.29)$ & $.74(.32)$ \\
Motivational orientation & $.08(.52)$ & $.10(.48)$ & $.15(.49)$ \\
Flossing at baseline & $4.16(3.69)$ & $2.97(3.29)$ & $3.31(3.08)$ \\
Flossing at one week & $5.69(3.76)$ & $5.38(3.74)$ & $4.83(2.82)$ \\
Recalled statements at baseline & $6.47(2.29)$ & $6.79(2.31)$ & $\mathrm{n} / \mathrm{a}$ \\
Recalled statements at one week & $3.56(2.82)$ & $3.36(2.52)$ & $\mathrm{n} / \mathrm{a}$ \\
\hline
\end{tabular}




\section{Table 2}

Hierarchical linear regression on self-reported flossing over one week by message frame, cultural exposure, and covariates.

\begin{tabular}{lll}
\hline $\boldsymbol{n}=\mathbf{1 3 0}$ & Step 1 & Step 2 \\
\hline & \multicolumn{2}{l}{ Unstandardized $\boldsymbol{B}(\boldsymbol{S E})$} \\
\hline Age & $-.17(.10)$ & $-.15(.10)$ \\
Female & $-.43(.57)$ & $-.40(.62)$ \\
African American & $.79(.72)$ & $.84(.75)$ \\
Asian American & $-.78(.93)$ & $-.83(.93)$ \\
Latino American & $.40(.81)$ & $.53(.83)$ \\
Past flossing & $.58(.08)^{* * *}$ & $.57(.08)^{* * *}$ \\
Loss frame & $4.46(1.61)^{* * *}$ & $4.43(1.62)^{* *}$ \\
Cultural exposure & $-4.98(1.58)^{* *}$ & $-4.79(1.60)^{* *}$ \\
Loss frame $\times$ Cultural exposure & $5.10(1.90)^{* *}$ & $4.97(1.92)^{* *}$ \\
Motivational orientation (MO) & & $-.66(.80)$ \\
Loss frame $\times$ MO & & $1.09(1.07)$ \\
\hline Note. & & \\
$* *$ \\
$p \leq 01$
\end{tabular}

